\title{
Histopathological Features of Group E Retinoblastoma Eyes after Upfront Enucleation
}

\author{
Muhammad Usman Viyani ${ }^{1}$, Jamal Mughal ${ }^{2}$, Muhammad Owais Arshad ${ }^{3}$, Anum Javed ${ }^{4}$, Javeria Nasir ${ }^{5}$, \\ Muhammad Hanif Chatni ${ }^{6}$ \\ ${ }^{1-6}$ Department of Ophthalmology, Patel Hospital, Karachi
}

\begin{abstract}
Purpose: To study histopathological features of eyes with intraocular Group E retinoblastoma primarily treated by enucleation at a tertiary care centre in Karachi, Pakistan.
\end{abstract}

Study Design: Retrospective histopathological study.

Place and Duration of Study: Department of Ophthalmology, Patel Hospital, Karachi, January 2012 to August 2019.

Methods: Seventy five eyes of children diagnosed with Group E retinoblastoma, on clinical examination, examination under general anaesthesia along with MRI and then enucleated were examined histopathologically after being primarily enucleated. Histological features were enlisted for the presence of choroidal invasion, extent of optic nerve invasion, anterior chamber involvement, scleral and extra-scleral invasion along with tumour differentiation. Demographic variables (age at presentation, gender, laterality of the disease, median follow-up) and the histopathological features of tumour along with high risk features were assessed using SPSS version 24.

Results: Out of 75 eyes, $48(64 \%)$ eyes showed high risk histopathological features including post lamina cribrosa involvement, choroidal invasion, scleral involvement, anterior chamber involvement (angle, iris, ciliary body). All high risk features were significantly more in the poorly differentiated group of tumours. All patients showing high risk features were given post enucleation systemic chemotherapy accordingly. Median follow-up was 40 months (3 - 100 months). Adjuvant chemotherapy was given in 48 (64\%) patients who showed high risk histopathological features.

Conclusion: By identifying the histopathological pattern and high risk features, we can decrease the chances of metastasis, recurrence, mortality and morbidity of these children which pose an overwhelming physical, psychological, social and financial burden on our society as a whole.

Key Words: Retinoblastoma, Enucleation, Chemotherapy.

How to Cite this Article: Viyani MU, Mughal J, Arshad MO, Javed A, Nasir J, Chatni MH. Histopathological Features of Group E Retinoblastoma Eyes after Upfront Enucleation. Pak J Ophthalmol. 2020; 36 (4): 329-334.

Doi: https://doi.org/10.36351/pjo.v36i4.1057

\section{INTRODUCTION}

It is well established that retinoblastoma is the most common primary intra-ocular tumour in young

Correspondence: Anum Javed

Department of Ophthalmology, Patel Hospital, Karachi

Email: anum.javed1989@gmail.com

Received: April 25, 2020

Accepted: July 23, 2020 children with worldwide prevalence of 1:15000 20000 live births. ${ }^{1}$ Pakistan ranks $6^{\text {th }}$ in the list of countries with high incidence of retinoblastoma. Around $66 \%$ of children are "diagnosed" before their second birthday according to World Health Organization (WHO), while 95\% are "diagnosed" before the age of five years. Presenting complaints of retinoblastoma include white reflex, red eye and proptosis, strabismus. ${ }^{2}$ Retinoblastoma may be unilateral or bilateral. A mutation in the RB1 gene 
holds responsible for both heritable and non-heritable forms. ${ }^{3}$ In spite of good understanding about the clinico-pathological features of retinoblastoma, the survival rate in Asia is comparatively low. ${ }^{4}$ Majority of the tumours are diagnosed at an advanced stage and those with metastatic retinoblastoma die within 6 months of time. ${ }^{5}$ The International Intraocular Retinoblastoma Classification (IIRC) is used for grouping of retinoblastoma. According to the IIRC, Group E eyes are destined for enucleation despite significant advancements in the treatment modalities available.

Histopathology shows the characteristics of the tumour, growth pattern and the extent of invasion by tumour. The histopathology of enucleated eyes serves to be an indicator for malignant potential of the tumour, including higher chances of orbital recurrence and metastasis. ${ }^{6}$ There is lack of general consensus on the identified high risk histopathology features among several researchers of enucleated eyes across the globe. ${ }^{7}$ However, residual tumour at the cut end of the optic nerve is unanimously agreed upon as a high risk feature.

With this study, the authors wish to share the histopathology features of Group E eyes from this part of the world and establish a relationship between age, tumour differentiation and high risk features.

\section{METHODS}

This is a retrospective study of 75 eyes that were diagnosed as group E Retinoblastoma on clinical examination, examination under general anaesthesia along with MRI. Approval from Ethical review board was sought. Informed consent was taken from the parents and the eyes were enucleated primarily at Patel Hospital, a tertiary care centre, in Karachi, Pakistan from January, 2012 to August, 2019. Files and histopathological documents were reviewed by the investigators. All slides were reviewed for tumour differentiation and high risk features. Their age, gender, laterality of the tumour, age at presentation and age at enucleation were noted. Median follow-up, number of deaths and extra-ocular metastasis with or without the presence of secondary tumours was also recorded. Histological features were enlisted for the presence of choroidal invasion, extent of optic nerve invasion, anterior chamber involvement, scleral and extra-scleral invasion along with tumour differentiation.
Eyes that were pathologically proven as intraocular retinoblastoma Group E and treated with enucleation as their primary treatment without any other local therapy, chemotherapy or radiation therapy, were included in the study. Eyes which had any other IIRC group or eyes that received treatment other than enucleation such as local laser treatment (Cryotherapy, Argon Laser), chemotherapy and radiation therapy before undergoing enucleation were excluded from the study. We performed enucleation only for eyes that were Group E according to IIRC.

High risk histopathological features were defined as choroidal invasion, invasion of the lamina cribrosa, tumour at the cut-end of the optic nerve, scleral infiltration and involvement of the anterior chamber by the tumour. Choroidal invasion is the term used for tumour cells invading the choroid with resultant compressive effects and infiltrative borders. Similarly, the extent of optic nerve involvement was grouped as no optic nerve invasion, pre-laminar optic nerve invasion, and post-laminar optic nerve invasion.

Level of differentiation was categorized as welldifferentiated to moderately differentiated tumours (comprising of small, circular, blue cells, showing features of differentiation in the form of flourettes, Flexner-Wintersteiner or Homer-Wright rosettes, staining with Synaptophysin), and as poorly differentiated tumours (tumours that had small, circular blue cells but no features of rosettes formation).

Adjuvant chemotherapy (Carboplatin, Etoposide, and Vincristine) was planned for those enucleated patients, who had high risk histopathological features such as optic nerve involvement posterior to lamina cribrosa, massive choroidal invasion along with any gross choroidal and optic nerve involvement and tumour seeding in anterior chamber or neovascular glaucoma.

SPSS Version 24 descriptive statistics were used to summarize the data with mean \pm standard deviation or mean for quantitative variables, taking P-value $<0.05$ as statistically significant. Frequencies and percentages were tabulated for categorical variables.

\section{RESULTS}

There were $50(66.7 \%)$ males and $25(33.3 \%)$ females. Median age at the time of presentation was 24 months (mean 26 months, range 3 to 72 months). Median age 
at the time of enucleation was 24 months (mean 27 months, range 3 to 73 months). Details are shown in table 1 .

Table 1: Demographics, Presenting Complains and Follow-up.

\begin{tabular}{lc}
\hline Demographics & n (Percentages) \\
\hline Number of patients & 75 \\
Number of eyes & 75 \\
Family History Positive & $10(13.3 \%)$ \\
Males & $50(66.7 \%)$ \\
Females & $25(33.3 \%)$ \\
Laterality & \\
Bilateral & $11(14.7 \%)$ \\
Unilateral & $64(85.3 \%)$ \\
Age & $\mathbf{n}($ Months $)$ \\
Median age at Presentation & 24 months \\
& $(3-72$ months) \\
Median age at Enucleation & 24 Months \\
& $(3-73 \mathrm{Months})$ \\
Presentation Signs & $\mathbf{n}($ Percentages) \\
Leukocoria & $55(73.3 \%)$ \\
Squint & $12(16 \%$ \\
Proptosis & $6(8 \%)$ \\
Hyphema & $2(2.7 \%)$ \\
ICRB Group: & \\
Group E & 75 \\
Grades of Differentiation & \\
Well-Differentiated & \\
Moderately-Differentiated & $23(30.7 \%)$ \\
Poorly Differentiated & $27(36)$ \\
Growth Pattern & $25(33.3)$ \\
Endophytic & \\
Exophytic & $47(62.7 \%)$ \\
Mixed & $22(29.3 \%)$ \\
Necrosis and Calcification & $6(8 \%)$ \\
Optic Nerve Cut End Not Involved & $47(62.7 \%)$ \\
Fotastasis or Death & $26(34.7 \%)$ \\
& 0 \\
& 40 \\
\hline &
\end{tabular}

The most prevalent high risk features in our study were choroidal involvement. Other features are depicted in table 2 .

No one received external beam radiotherapy (EBRT). Median follow-up was 40 months $(3-100$ months). Adjuvant chemotherapy was given in 48 (64\%) patients who showed high risk histopathological features. No evidence of tumour metastasis, recurrences or deaths were recorded for any patient during the study period.
Table 2: High Risk Features on Histopathology of Well/ Moderately Differentiated and Poorly Differentiated Tumours.

\begin{tabular}{lcc}
\hline High Risk Features & $\begin{array}{c}\text { Well/Moderately } \\
\text { Differentiated } \\
\text { n(Percentages) }\end{array}$ & $\begin{array}{c}\text { Poorly } \\
\text { Differentiated } \\
\text { n (Percentages) }\end{array}$ \\
\hline $\begin{array}{l}\text { Optic Nerve Cut End } \\
\text { Involved }\end{array}$ & $18(36 \%)$ & $8(32 \%)$ \\
Post Lamina Cribrosa & $19(38 \%)$ & $11(44 \%)$ \\
$\begin{array}{l}\text { Involvement } \\
\text { Choroidal Invasion }\end{array}$ & $28(56 \%)$ & $18(72 \%)$ \\
Scleral Involvement & $13(26 \%)$ & $9(36 \%)$ \\
Anterior Segment & & \\
Involvement & $5(10 \%)$ & $4(16 \%)$ \\
$\quad$ Angle & $3(6 \%)$ & $5(20 \%)$ \\
$\quad$ Iris & $1(2 \%)$ & $3(12 \%)$ \\
$\quad$ Ciliary Body & $14(28 \%)$ & $12(48 \%)$ \\
\hline
\end{tabular}

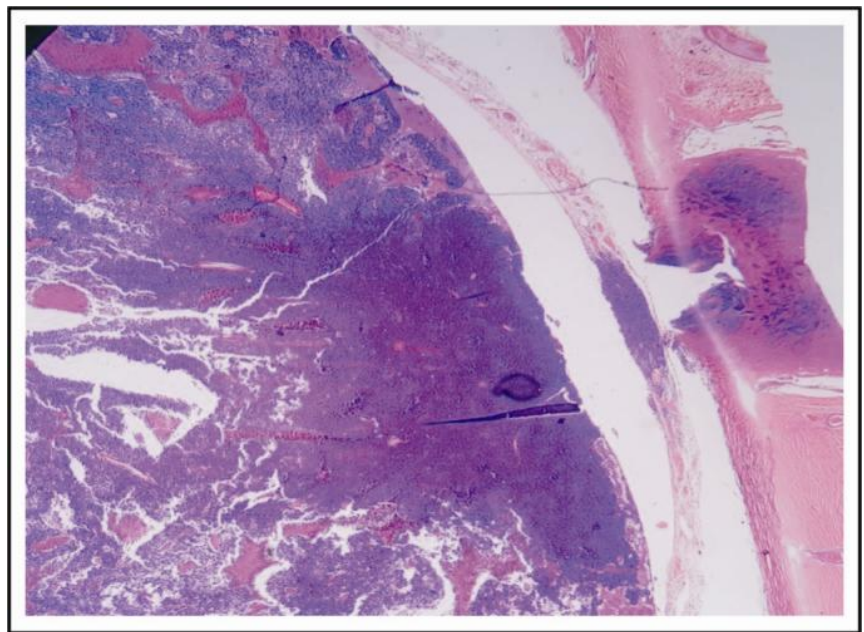

Fig. 1: Specimen Showing Pre-laminar Optic Nerve Invasion.

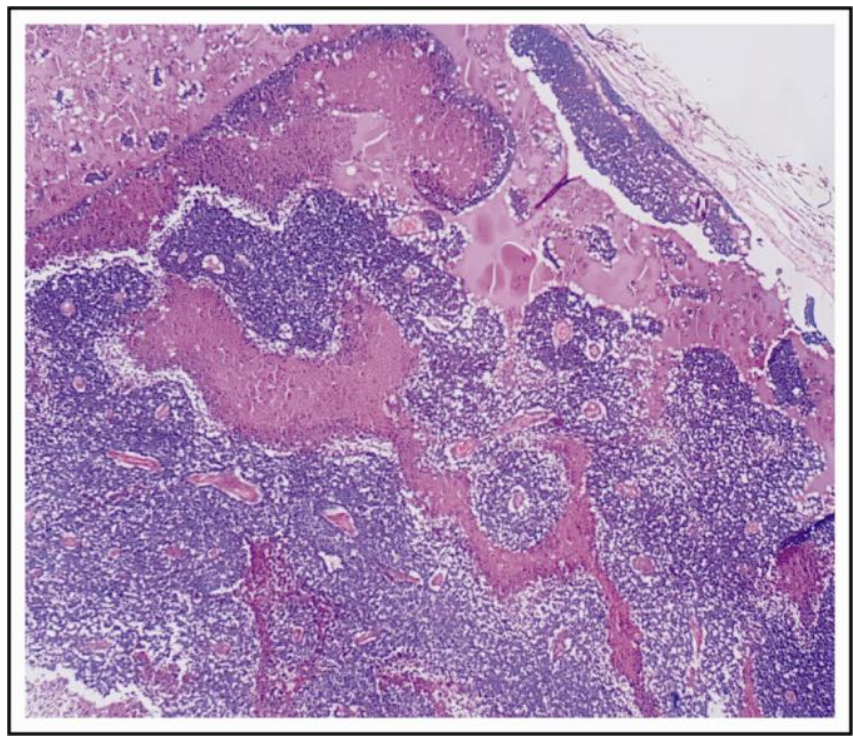

Fig. 2: Specimen Showing Homer Wright Rosettes and Flexner Wintersteiner Rosettes. 


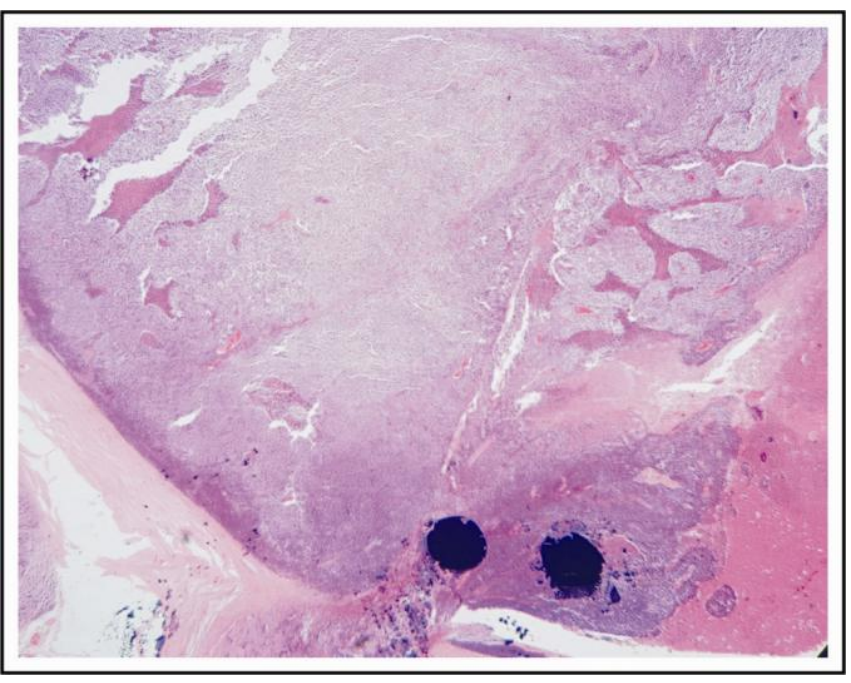

Fig. 3: Specimen Showing Necrosis and Few Calcifications.

\section{DISCUSSION}

Retinoblastoma can often lead to death in children if the disease is left untreated. Outcomes of retinoblastoma vary among the developed and developing countries. In countries like Pakistan, delay in seeking specialist health care and refusal of treatment further leads to higher mortality. Hindrances encountered while handling the histopathological specimens and an avid lack of awareness about the disease further add to the overall disease burden. This delay in seeking medical and/or ophthalmological advice could be a possible cause of increased chances of retinoblastoma when compared to the developed countries including Europe and North America.

In this study we reviewed the records of 75 enucleated eyes of retinoblastoma patients. We found high risk histopathological features in 46 eyes postenucleation. A precise definition of high risk features has been argued and disputed variably but an acceptable one includes gross choroidal invasion with post-laminar optic nerve involvement, tumour present at the cut end of the optic nerve and scleral invasion. These are the features that are associated with a high risk of metastatic disease and require further treatment. ${ }^{8}$ Various studies have been conducted on the incidence and implications of high risk histopathological features worldwide. $10-40 \%$ of advanced intraocular disease (group D and group E) is suggested to be associated with high risk histopathology at diagnosis. Post-laminar optic nerve invasion and massive choroidal invasion constitute the most common features, similar to the findings of Kim and colleagues. ${ }^{9}$

The median age of diagnosis was 24 months which was consistent with the findings of other researchers from subcontinent. Subramaniam et al found that the mean age at diagnosis was between $23-24$ months of age whereas in the western literature, mean age at diagnosis is reported as 18 months. ${ }^{10}$ Our results concluded that irrespective of laterality, poorly differentiated cases presented late, compared to those that were well to moderately differentiated, which were similar to the works of Eagle et $\mathrm{al}^{11}$ and Madhavan et al. ${ }^{12}$ Necrosis was found along with calcification in $47(62.7 \%)$ cases. It was observed in our study that the more the necrosis in the tumour, the higher the chance of invasion beyond the lamina cribrosa of the optic nerve. Histopathological high risk features in our group of patients, were found to be similar to other studies from India. ${ }^{13}$ The reported incidence of choroidal invasion varies from $12 \%$ to $41 \%$ while optic nerve involvement with resection end range from $6.5 \%$ to $40 \%$, discussed by Yousef et al. ${ }^{14}$ Similarly, current study showed majority $(61.4 \%)$ of the eyes had choroidal invasion.

In our study, younger patients were found to have well-differentiated tumours in contrast to older patients, which are often associated with poorly differentiated tumours. There were low to intermediate features of histopathology in younger patients as opposed to those who were enucleated at an older age (> 2.5 years). These findings were consistent with the study of Kaliki et al, suggesting that welldifferentiated tumours present earlier than poorly differentiated tumours. This may be related to varying retinal cell affection with development and dedifferentiation of tumours with age. ${ }^{15}$

There was no evidence of extraocular disease on MRI prior to enucleation. Histopathology remains the gold standard in identifying high risk features. However, De Jong et al studied the significance of MRI addressing the high risk features. Identification of these features on MRI assists greatly in making the choice of enucleation versus globe-salvage therapy. It is believed that MRI findings should not, however, be the basis of taking the final decision of enucleation. Hence, it is suggested that vigilant and thorough screening prior to the surgical procedure of enucleation should be employed. ${ }^{16}$

None of the patients received any form of treatment before enucleation. As studied by Zhao et al, 
the authors believe that chemotherapy offered before enucleation to Group E eyes with advanced tumour, may alter the pathological features and down stage the disease. ${ }^{17}$ This can have a masking effect on the possible risk of metastasis and even death.

More than half of the eyes were well to moderately differentiated while the rest were poorly differentiated. This could partly be explainable due to the early decision for upfront enucleation of group $\mathrm{E}$ eyes in our setup. However, in our neighbouring countries, as discussed by Kashyap et al, most of the tumours were poorly differentiated, owing to late presentation and delayed referral among medical personals. $^{18}$

During our study period, there was no evidence of metastasis, recurrence of disease or deaths recorded due to the fact that they were given timely chemotherapy. Moreover, this could be due to almost similar age at presentation and prompt decision for upfront enucleation based in our results. The epitome of successful retinoblastoma treatment revolves around saving the life of the patient, decreasing and/or eliminating the risk of metastasis, salvaging the globe and vision, along with an overall improvement in the quality of life of those afflicted with this debilitating and fatal disease. ${ }^{19}$

Various researches across the globe have debated over high risk features from developed and developing countries. However, there are very few studies available from Pakistan about high risk features of retinoblastoma afflicted Group E enucleated eyes. ${ }^{20}$

The limitation of our study was the limited number of cases studied. To establish a consensus, we need further multi-centre studies with larger sample size, which may offer a better understanding and better insight about histopathological trends of retinoblastoma in Pakistan. There is scarcity of published data from our part of the world.

\section{CONCLUSION}

By identifying the high grade histopathological features we can manage and devise a strategy to deal with this unprecedented disease.

\section{Ethical Approval}

The study was approved by the Institutional review board/Ethical review board.

\section{Conflict of Interest}

Authors declared no conflict of interest.

\section{REFERENCES}

1. Jain M, Rojanaporn D, Chawla B, Sundar G, Gopal L, Kheta V. Retinoblastoma in Asia. Eye, 2019; 33 (1): 87-96.

2. Zia N, Hamid AA, Iftikhar S, Qadri MH, Jangda A, Khan MR. Retinoblastoma Presentation and Survival: A four-year analysis from a tertiary care hospital. Pak J Med Sci. 2020; 36 (1): S61.

3. Nawaiseh I, Al-Hussaini M, Alhamwi A, Meyar M, Sultan I, Alrawashdeh K, et al. The Impact of Growth Patterns of Retinoblastoma (Endophytic, Exophytic, and Mixed Patterns). J. Pathol. 2015; 31 (1): 45-50.

4. Kivelä T. The epidemiological challenge of the most frequent eye cancer: retinoblastoma, an issue of birth and death. Br J Ophthalmol. 2009; 93 (9): 1129-1131. Doi:https://doi.org/10.1136/bjo.2008.150292.

5. Dimaras H, Kimani K, Dimba EA, Gronsdahl P, White A, Chan HS, et al. Retinoblastoma. The Lancet. 2012; 379 (9824): 1436-1446.

6. Singh L, Kashyap S. Update on pathology of retinoblastoma. Int. J. Ophthalmol. 2018; 11 (12): 2011.

7. Aerts I, Sastre-Garau X, Savignoni A, Lumbroso-Le Rouic L, Thebaud-Leculée E, Frappaz D, et al. Results of a multicenter prospective study on the postoperative treatment of unilateral retinoblastoma following primary enucleation. J Clin Oncol. 2013; 31: 1458-1463.

8. Chévez-Barrios P, Eagle Jr RC, Krailo M, Piao J, Albert DM, Gao Y, et al. Study of Unilateral Retinoblastoma with and without Histopathologic High-Risk Features and the Role of Adjuvant Chemotherapy: A Children's Oncology Group Study. J. Clin. Oncol. 2019; 37 (31): 2883-2891.

9. Kim JW, Shah SN, Green S, O'Fee J, Tamrazi B, Berry JL. Tumour size criteria for Group D and E eyes in the International Classification System for Retinoblastoma: effects on rates of globe salvage and high-risk histopathologic features. Acta Ophthalmologica, 2020; 98 (1): e121-125.

10. Subramaniam S, Rahmat J, Rahman NA, Ramasamy S, Bhoo-Pathy N, Pin GP, et al. Presentation of retinoblastoma patients in Malaysia. Asian Pac J Cancer Prev. 2014; 15 (18): 7863-7867.

11. Eagle Jr RC. High-risk features and tumor differentiation in retinoblastoma: a retrospective histopathologic study. Arch. Pathol. Lab. Med. 2009; 133 (8): 1203-1209.

12. Madhavan J, Ganesh A, Roy J, Biswas J, Kumaramanickavel G. The relationship between tumor cell differentiation and age at diagnosis in retinoblastoma. J Pediat Ophth Strab. 2008; 45 (1): 2225. 
13. Kashyap S, Meel R, Pushker N, Sen S, Bakhshi S, Sreenivas $\mathbf{V}$, et al. Clinical predictors of high risk histopathology in retinoblastoma. Pediatr Blood Cancer, 2012; 58 (3): 356-361.

14. Yousef YA, Hajja Y, Nawaiseh I, Mehyar M, Sultan I, Deebajah R, et al. A Histopathologic Analysis of 50 Eyes Primarily Enucleated for Retinoblastoma in a Tertiary Cancer Center in Jordan. J. Pathol. 2014; 30 (3): 171-177.

15. Kaliki S, Gupta S, Ramappa G, Mohamed A, Mishra DK. High-risk retinoblastoma based on age at primary enucleation: a study of 616 eyes. Eye, 2019; 25: $1-8$.

16. De Jong MC, van der Meer FJ, Göricke SL, Brisse HJ, Galluzzi P, Maeder P, et al. Diagnostic accuracy of intraocular tumor size measured with MR imaging in the prediction of postlaminar optic nerve invasion and massive choroidal invasion of retinoblastoma. Radiology, 2016; 279 (3): 817-826.

17. Zhao J, Dimaras H, Massey C, Xu X, Huang D, Li BChan HS, et al. Pre-enucleation chemotherapy for eyes severely affected by retinoblastoma masks risk of tumor extension and increases death from metastasis. J Clin Oncol. 2011; 29 (7): 845-851.

18. Kashyap S, Sethi S, Meel R, Pushker N, Sen S, Bajaj MS, et al. A histopathologic analysis of eyes primarily enucleated for advanced intraocular retinoblastoma from a developing country. Arch. Pathol. Lab. Med. 2012; 136 (2): 190-193.

19. Kaliki S, Mittal P, Mohan S. Chattannavar G, Divya S, Jajapuram, et al. Bilateral advanced (group D or E) intraocular retinoblastoma: outcomes in 72 Asian Indian patients. Eye, 2019; 33: 1297-1304. https://doi.org/10.1038/s41433-019-0409-z
20. Khan A, Bukhari M, Mehboob R. Association of retinoblastoma with clinical and histopathological risk factors. Natural Science. 2013; 5: 437-444.

Doi: https://doi.org/10.4236/ns.2013.54056.

\section{Authors' Designation and Contribution}

Muhammad Usman Viyani; Consultant Ophthalmologist: Concepts, Design, Literature research, Statistical Analysis, Manuscript editing, Manuscript review.

Jamal Mughal; Consultant Ophthalmologist: Concepts, Design, Manuscript editing, Manuscript review.

Muhammad Owais Arshad; Senior Registrar: Literature research, Data Acquisition, Data Analysis, Statistical Analysis, Manuscript editing, Manuscript review.

Anum Javed; Post Graduate Trainee: Literature research, Data Acquisition, Data Analysis, Statistical Analysis, Manuscript editing, Manuscript review.

Javeria Nasir; Registrar: Literature research, Data Acquisition, Data Analysis, Statistical Analysis, Manuscript editing, Manuscript review.

Muhammad Hanif Chatni; Director Eye Department: Concepts, Design, Manuscript preparation, Manuscript editing, Manuscript review. 